\title{
Freqüência das alterações of talmológicas em pacientes com hanseníase residentes em hospital-colônia
}

\author{
Frequency ofocularchanges in patients with Hansen's diseaseliving in a colony hospital
}

\author{
Francineide Sadala de Souza ${ }^{1}$ \\ Luciana Negrão Frota de Almeida ${ }^{2}$ \\ Joângela Pereira Costa ${ }^{3}$ \\ Patrícia Valiati da Rocha ${ }^{4}$ \\ Edmundo Frota de Almeida Sobrinho ${ }^{5}$
}

\begin{tabular}{|l|}
\hline RESUMO \\
\hline Objetivos: Identificar a frequiência das alterações oculares em pacientes \\
hansenianos residentes em hospital-colônia; comprovar a predileção pelo \\
segmento anterior do olho em relação ao posterior. Métodos: Fez-se estudo \\
transversal de 115 olhos de 58 pacientes internados no abrigo João Paulo II, \\
em Marituba - Pará, no período de agosto a outubro de 1999. Os dados \\
epidemiológicos da pesquisa foram obtidos da ficha de cada paciente e de \\
dados colhidos durante o exame oftalmológico. Todos os pacientes estu- \\
dados estavam curados da hanseníase segundo as normas do Ministério da \\
Saúde. Resultados: Em 114 olhos (99,1\%) foi observado envolvimento \\
ocular, sendo a maioria (77,2\%) pertencente à forma virchowiana. Os \\
achados oculares mais freqüentes nos anexos oculares foram a madarose \\
ciliar parcial (70,4\%) e a madarose superciliar parcial (59,1\%). Quanto ao \\
bulbo ocular, o achado mais freqüente relacionado à hanseníase foi a \\
diminuição e/ou ausência de sensibilidade corneana observada em $42,6 \%$ \\
dos olhos, seguida da midríase paralítica (16,5\%) encontrada no pós- \\
operatório dos pacientes submetidos a facectomia, achado ligado intima- \\
mente à atrofia de íris (8,7\%), comumente observada nestes pacientes. Não \\
foi encontrada nenhuma alteração à fundoscopia que pudesse ser atribuída \\
à hanseníase. Conclusões: A alta prevalência de alterações do bulbo ocular \\
e anexos em pacientes portadores de hanseníase, principalmente na forma \\
virchowiana, indicam a necessidade da assistência contínua a esses pa- \\
cientes mesmo após a cura da doença.
\end{tabular}

Descritores: Hanseníase; Hanseníase virchowiana; Mycobacterium leprae; Manifestações oculares; Infecções oculares bacterianas; Oftalmopatias/prevenção \& controle; Hospitais; Estudos transversais
Trabalho realizado na Colônia de Marituba (PA) - Abrigo João Paulo II - com o apoio do Centro de Referência Dermatológica Dr. Marcelo Cândia.

Graduada em Medicina pela Universidade Federal do Pará (UFPA). Belém (PA).

${ }^{2}$ Acadêmica do $6^{\circ}$ ano de Medicina da Universidade Estadual do Pará (UEPA). Belém (PA).

${ }^{3}$ Graduada em Medicina pela UFPA. Belém (PA)

${ }^{4}$ Graduada em Medicina pela UFPA. Belém (PA).

${ }^{5}$ Oftalmologista do Centro de Referência em Dermatologia Sanitária do Instituto Marcelo Cândia. Doutor em Oftalmologia pela Clínica Oftalmológica da Universidade Federal de Minas Gerais (UFMG). Belo Horizonte (MG). Professor de Oftalmologia da UEPA. Belém (PA).

Endereço para correspondência: Edmundo Frota de Almeida Sobrinho - Rua Conselheiro Furtado, 2818 Ap. 14000 - Belém (PA) CEP 66063-060

E-mail:ealmeida@amazon.com.br

Recebido para publicação em 12.07.2002

Versão revisada recebida em 06.12.2004

Aprovação em 27.01.2005

Os autores declaram que não possuem interesse financeiro no desenvolvimento do estudo.

\section{INTRODUÇ̃̃O}

O Mycobacterium leprae é uma bactéria gram positiva, que lesa a pele e os nervos periféricos, tendo predileção pelas áreas mais frias do corpo (nariz, testículos, pavilhão auditivo, olhos e outros órgãos) determinando uma doença crônica denominada hanseníase ${ }^{(1-4)}$.

Particularmente no aparelho visual, determinam lesões tanto nos anexos oculares quanto no bulbo ocular, levando aos mais variados graus de incapacidade visual ${ }^{(1-4)}$. Tais lesões são decorrentes da agressão direta ou indireta do bacilo às estruturas oculares, sendo o mecanismo indireto representado pelos processos reacionais ${ }^{(4)}$. Nos anexos oculares são representadas pela madarose, triquíase, ectrópio, queda do supercílio, blefarocálases, lagoftalmo e diminuição da secreção lacrimal, ao passo que no bulbo ocular os principais achados são a alteração da sensibilidade e da transparência da córnea, ceratites, esclerites, iridociclites, dentre outras. 
$\mathrm{O}$ fato das lesões nervosas atingirem os membros superiores e inferiores, causando deformidades, atrofias e úlceras, terminam por incapacitar o indivíduo. A visão, torna-se, portanto, um indispensável sentido para inspecionar suas lesões e para permitir o melhor relacionamento com o ambiente ${ }^{(1,5)}$.

\section{MÉTODOS}

Foram examinados 115 olhos e 116 anexos oculares de 58 pacientes hansenianos internados no Abrigo João Paulo II, em Marituba-PA, sendo que uma paciente da forma virchowiana apresentava enucleação prévia de um olho. Os pacientes foram submetidos ao exame oftalmológico no período de agosto a outubro de 1999 e todos já haviam recebido alta por cura, segundo as normas do Ministério da Saúde.

A coleta de dados epidemiológicos e dermatológicos foi realizada nos prontuários dos pacientes e registradas nas fichas de avaliação oftalmológica utilizadas para a realização deste estudo.

O exame oftalmológico obedeceu a um protocolo pré-determinado pelos autores, que consistia em: identificação do paciente, interrogatório dirigido dos principais sintomas relacionado à doença, ectoscopia, teste de sensibilidade corneana, medida da acuidade visual, biomicroscopia do segmento anterior e posterior, oftalmoscopia binocular direta e indireta e tonometria de aplanação. A forma clínica da doença só foi identificada ao final do exame para não interferir nos resultados.

\section{RESULTADOS}

Na população estudada, 41 pacientes $(70,7 \%)$ pertenciam ao sexo masculino e apenas $17(29,3 \%)$ ao sexo feminino. A idade dos pacientes variou de 30 a 90 anos.

Em 115 olhos estudados, $89(77,4 \%)$ pertenciam a pacientes da forma virchowiana, $14(12,2 \%)$ da forma tuberculóide e $12(10,4 \%)$ da forma dimorfa. Um paciente com a forma virchowiana apresentou enucleação prévia de um olho. Não foi encontrado nenhum paciente do grupo Indeterminado.

A presença de alterações oculares e/ou de seus anexos foi observada em 114 olhos, sendo $88(77,2 \%)$ pertencentes a pacientes da forma virchowiana, $14(12,3 \%)$ da forma tuberculóide e $12(10,5 \%)$ da forma dimorfa.

O sintoma mais freqüente, independente da forma clínica, foi o prurido ocular observado em 59 olhos $(51,3 \%)$ seguido de baixa acuidade visual em $57(49,5 \%)$, ardor e lacrimejamento em $55(47,8 \%)$, fotofobia em $37(32,1 \%)$, dor em $36(31,3 \%)$ e secreção em $33(28,7 \%)$.

Anexos oculares: A madarose ciliar parcial foi encontrada em 81 olhos $(70,4 \%)$ seguidas de madarose superciliar parcial observada em $68(59,1 \%)$, ptose ciliar em 31 (26,9\%), queda de supercílios em $22(19,1 \%)$. A blefarocálase, a madarose ciliar total e a dificuldade de drenagem da lágrima foram observadas em 19 olhos $(16,5 \%)$ enquanto que a madarose superciliar total foi encontrada em $18(15,6 \%)$. Em ordem decrescente de freqüência, verificou-se a triquíase e o ectrópio de pálpebra inferior encontradas em 15 olhos (13,0\%), a distiquíase em 10 $(8,6 \%)$, o entrópio em $5(4,3 \%)$, as infiltrações de face em 4 $(3,4 \%)$, o entrópio de pálpebra inferior em $3(2,6 \%)$. O lagoftalmo ocorreu em 17 olhos $(14,8 \%)$ com maior frequiência na forma virchowiana onde foram encontrados 9 olhos $(52,9 \%)$. Não foi observado nenhum caso de hansenoma.

A dificuldade de drenagem da lágrima detectada pelo teste de Milder em $19(16,5 \%)$ casos esteve associada ao lagoftalmo em 3 casos (15,8\%), ao ectrópio de pálpebra inferior em 4 casos $(21,0 \%)$ e ambos os casos em 9 olhos $(47,3 \%)$ (Gráfico 1).

Bulbo ocular: A presença do pterígio foi observada em 29 olhos $(25,2 \%)$, hiperemia conjuntival foi verificada em 13 olhos $(11,3 \%)$ e a presença de secreção em $5(4,3 \%)$. O estafiloma foi observado em 3 olhos (2,6\%), todos de pacientes portadores da forma virchowiana, e se constituiu no principal achado escleral. $\mathrm{O}$ adelgaçamento da esclera foi identificado em 2 olhos $(1,73 \%)$. Não foi encontrado nenhum caso de esclerite.

A hipoestesia corneana foi observada em 36 olhos $(31,3 \%)$ e a anestesia em 13 deles $(11,3 \%)$. A úlcera de córnea foi diagnosticada em 8 olhos $(6,9 \%)$, desses, 4 (50\%) pertenciam a pacientes da forma virchowiana e $4(50 \%)$ do grupo Dimorfo. Em 19 olhos (16,5\%) foi observada a presença de opacidade corneana com predomínio do leucoma sobre as demais. A presença de vasos na córnea foi verificada em 9 olhos $(7,8 \%)$.

$\mathrm{O}$ encontro de "flare" na câmara anterior foi irrelevante, evidenciado apenas em um olho $(0,8 \%)$ da forma virchowiana. Células na câmara anterior não foram observadas nos pacientes examinados.

Na pupila, a midríase paralítica foi à alteração mais freqüente verificada em 19 olhos (16,5\%), sendo que em $13(68,4 \%)$ estavam relacionadas a facectomia anterior e em $6(31,6 \%)$ essas associação não foi observada. A miose foi diagnosticada em apenas 5 pacientes $(4,3 \%)$.

$\mathrm{Na}$ íris, a atrofia de estroma encontrada em 10 olhos $(8,7 \%)$ foi a alteração mais expressiva do trato uveal. Em 6 olhos $(5,2 \%)$ observou-se atrofia do estroma e do epitélio pigmentado da íris. Sinéquias posteriores foram observadas em 4 olhos $(3,5 \%)$, sendo $2(50 \%)$ desses em pacientes portadores da forma virchowiana e $2(50 \%)$ em portadores da forma tuberculóide. Catarata senil foi observada em 50 olhos $(43,5 \%)$ e em 32 olhos $(27,8 \%$ ) pseudofacia (Gráfico 2).

Fundoscopia: A escavação glaucomatosa foi observada em 10 olhos $(8,7 \%)$. Este exame foi prejudicado em 12 olhos (10,4\%), sendo que em $8(66,6 \%)$ devido à presença de leucoma, $3 \mathrm{em}$ (25\%) devido à catarata e em $1(8,3 \%)$ olho cego. Degeneração macular relacionada à idade (DMRI) foi observada em 16 olhos $(13,9 \%)$ e descolamento posterior de vítreo em 6 olhos $(5,2 \%)$. Não foi detectada nenhuma alteração do segmento posterior do olho que pudesse ser atribuída à hanseníase.

Tonometria: A pressão intra-ocular (PIO) esteve superior a $20 \mathrm{mmHg}$ em apenas 4 olhos $(3,5 \%)$ e esteve inferior a $12 \mathrm{mmHg}$ em 20 (17,39\%).

A acuidade visual (AV) em 31 olhos (26,9\%) foi de 0,4 ou melhor, em $18(15,6 \%)$ foi entre 0,3 e 0,2 , e em $34(29,5 \%)$ foi 


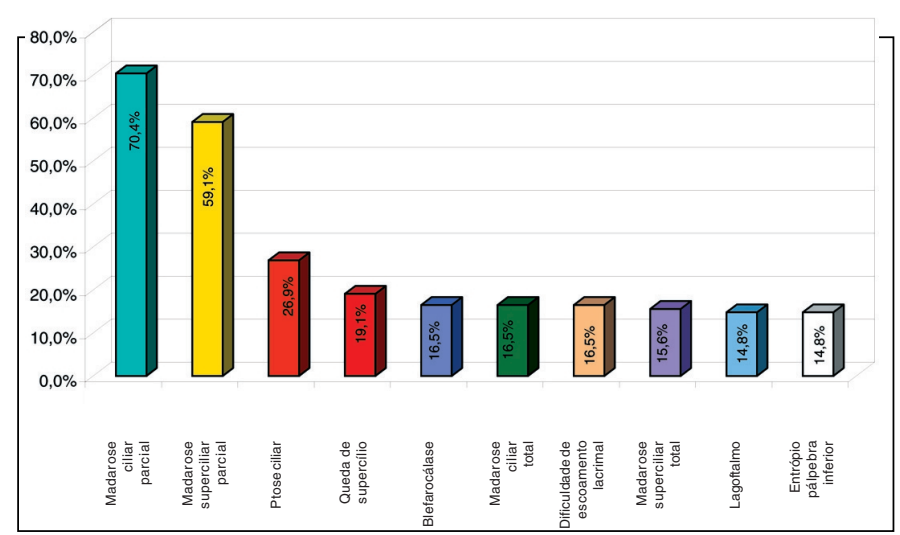

Gráfico 1 - Principais alterações de anexos oculares em pacientes com hanseníase do Abrigo João Paulo II - Marituba, Estado do Pará, 1999

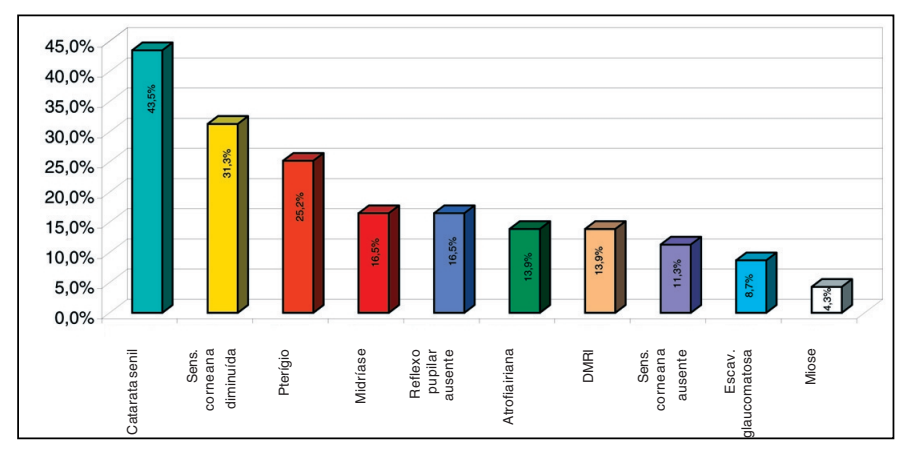

Gráfico 2 - Principais alterações do bulbo ocular em pacientes com hanseníase do Abrigo João Paulo II - Marituba, Estado do Pará, 1999

igual ou inferior a 0,1 . Apenas 8 olhos $(6,9 \%)$ apresentaram visão de vultos, $15(13,0 \%)$ visão de conta-dedos e em $7(6,0 \%)$ visão zero. $\mathrm{O}$ exame ficou prejudicado em 2 olhos $(1,7 \%)$ pela incapacidade de se obter informações do paciente.

Outros achados incluem a midríase encontrada em 11 olhos $(9,5 \%)$ sendo verificada em concomitância com a facectomia prévia em 10 olhos $(90,9 \%)$ e em 1 olho $(9,0 \%)$ com glaucoma; o pterígio foi observado em 29 (25,2\%); a catarata senil em 50 (43,5\%); a degeneração macular relacionada à idade (DMRI) em $16(13,9 \%)$ e o descolamento posterior de vítreo encontrado em 6 olhos $(5,2 \%)$.

Do total de olhos examinados, 32 (27,8\%) haviam sido submetidos previamente a facectomia com implante de lente intra-ocular.

\section{DISCUSSÃO}

A não ocorrência de pacientes da forma indeterminada na população estudada apóia-se no fato de que pacientes de hospital-colônia possuem longo tempo de duração da doença e por isso já tem uma forma clínica definida.

O levantamento do tempo de doença destes pacientes não foi estabelecido visto as dificuldades na obtenção desses dados, tanto diretamente dos pacientes, quanto de seus prontuários.
A predominância do sexo masculino $(70,6 \%)$ deve-se à maior exposição e contaminação pelo bacilo em nosso meio ${ }^{(3,6-8)}$. Enquanto que em algumas regiões do mundo, onde homens e mulheres possuem ocupação semelhante, tal diferença não é observada, podendo haver inclusive predomínio do sexo feminino ${ }^{(9)}$. Portanto, a ocupação pode ser fator favorecedor na epidemiologia da doença, podendo predispor à doença desde que associada a outros fatores importantes tais como os imunológicos, genéticos e sociais.

A AV igual ou inferior a 0,3 , incluindo visão de vultos e conta dedos, foi verificada em 75 olhos $(65,2 \%)$. O porcentual de perda de visão total nesta população foi de 6,0\% (7 olhos), dados inferiores aos encontrados na literatura ${ }^{(10)}$. Dentre estes, 6 pertenciam à forma Virchowiana $(85,7 \%)$ e apenas $1(14,3 \%)$ ao grupo dimorfo, demonstrando a maior agressividade da primeira forma clínica. Observou-se um caso de cegueira unilateral por acidente traumático, outro por acidente químico e outro por glaucoma em pacientes portadores da forma virchowiana.

A madarose ciliar parcial, seguida da madarose superciliar parcial e da ptose ciliar, foram as alterações mais frequientes dentre as estudadas nos anexos oculares, divergindo da literatura onde a madarose superciliar ${ }^{(3,7)}$ e a ptose ciliar $^{(11)}$ se constituiu no achado mais freqüente.

Neste estudo, o lagoftalmo ocorreu em $14,8 \%$ dos casos com maior freqüência na forma virchowiana $(50 \%)$ coincidindo com os relatos de vários autores, onde esta forma clínica predominou apesar de diferenças quanto à freqüência deste $\operatorname{achado}^{(3,7,10)}$.

O ectrópio foi encontrado em 17 olhos (14,8\%), porcentagens equivalentes desta alteração são descritas por alguns autores $^{(11)}$, mostrando a importância desse achado no quadro ocular da hanseníase. A blefarocálase foi encontrada em 19 olhos $(16,5 \%)$.

Em relação ao aparelho lacrimal constatou-se através do Teste de Milder, resultado positivo, denotando dificuldade de drenagem das lágrimas, em 19 olhos (16,5\%), sendo que em 16 $(13,9 \%)$ olhos essa alteração foi concomitante com a presença de ectrópio da pálpebra inferior e lagoftalmo.

As alterações do bulbo ocular foram encontradas em 82 olhos $(71,3 \%), 56(68,3 \%)$ da forma virchowiana, $12(14,6 \%)$ do grupo dimorfo e $14(17,0 \%)$ da forma tuberculóide, dados discordantes da maioria da literatura consultada ${ }^{(3,7-8,10)}$. Como por exemplo, um percentual de $45,6 \%$ de alterações bulbares em pacientes da forma virchowiana e apenas $2 \%$ nos pacientes da forma tuberculóide ${ }^{(7)}$.

$\mathrm{Na}$ conjuntiva, considerada uma das prováveis vias de penetração do bacilo no bulbo ocular ${ }^{(12)}$, observou-se pterígio em $25,2 \%$ dos casos, dados semelhantes aos de alguns autores ${ }^{(1)}$ que acreditam que este possa estar relacionado à faixa etária dos pacientes e à exposição ocular que estes experimentam no lagoftalmo. Tais alterações não são específicas da doença e não há nenhum relato da participação do $M$. leprae em sua gênese, apesar do achado do bacilo em esfregaços de raspados da conjuntiva tarsal, no saco lacrimal e conjuntiva bulbar ${ }^{(6,12)}$.

$\mathrm{Na}$ esclera, foi evidenciado a presença de estafiloma em $2,61 \%$ dos olhos, todos pertencentes à forma virchowiana, não sendo encontrado nenhum caso de esclerite. 
Foram diagnosticados 49 casos $(42,6 \%)$ de sensibilidade corneana diminuída ou ausente, achados concordantes com vários autores ${ }^{(2,9-10)}$.

A ausência de células na câmara anterior e a observação de apenas um caso de "flare" reforçam a idéia de que a uveíte não é freqüente neste tipo de população e tampouco é a causa principal de cegueira como habitualmente é citado na literatura ${ }^{(3,5,7,10)}$.

A perda do reflexo pupilar foi a alteração mais freqüente no exame da pupila, encontrada em 19 casos (16,5\%) estando relacionado diretamente com a facectomia prévia a que foram submetidos esses pacientes em 13 olhos $(68,4 \%)$. Do mesmo modo que contribuiu para o encontro de midríase em 12 casos $(10,4 \%)$, achado incomum, uma vez que pupilas normais ou mióticas são as que habitualmente se encontram nos portadores de hanseníase ${ }^{(1,3,5)}$. O único caso de midríase que não pôde ser explicado pelo procedimento cirúrgico prévio, foi observado em um olho cego por glaucoma em uma paciente portadora da forma virchowiana.

Foram observados 10 casos de atrofia iriana $(8,7 \%)$ e esta foi a principal alteração observada nesta estrutura, o mesmo foi constatado por diversos autores ${ }^{(3,13-14)}$. Estes autores acreditam que a atrofia iriana seja decorrente de um processo degenerativo, compreendido no passado como um processo inflamatório, idéia que até hoje é encontrada nos compêndios de hanseníase.

A catarata é um achado comum nas colônias de hansenianos principalmente pela faixa etária desses pacientes ${ }^{(3)}$. Neste estudo, foram verificados 50 casos $(43,5 \%)$ de catarata, enquanto que em alguns trabalhos verificou-se a prevalência da catarata de $24,9 \%$ em pacientes hansenianos ${ }^{(9)}$. Muitos autores acreditam que a inflamação da íris determinada pela doença acelere o processo de opacificação do cristalino $^{(9,13)}$.

Os achados no segmento posterior são raros e, apesar do isolamento do bacilo no corpo vítreo e na retina periférica, este não parece estar relacionado às alterações fundoscópicas por vezes encontradas ${ }^{(3,6-7,9)}$. A degeneração macular relacionada à idade, apesar de não ter relação com a hanseníase, foi a alteração mais encontrada, presente em 13,9\% dos casos, seguida da escavação do disco óptico aumentada em 10 olhos $(8,7 \%)$. Dentre os olhos que apresentaram escavação do disco óptico aumentada, cerca de $40 \%$ apresentavam pressão intraocular menor que $20 \mathrm{mmHg}$.

\section{CONCLUSÃO}

O elevado número de alterações observadas tanto no bulbo ocular como nos anexos oculares em portadores de hanseníase, sobretudo na forma virchowiana, são responsáveis pela diminuição de visão, sentido de demasiada importância principalmente para os pacientes que apresentam alto grau de incapacidade física. Isto serve de alerta para que seja instituído o exame ocular na rotina de atendimento desses pacientes, visto que o diagnóstico e tratamento precoces previnem as graves manifestações da hanseníase ocular.

\section{ABSTRACT}

Purpose: To identify the frequency of ocular changes in patients with Hansen's disease, living in a colony hospital; to confirm the predilection for the anterior segment of the eye instead of the posterior segment. Methods: Transversal study of 115 eyes of 58 in-patients at João Paulo II colony, Marituba Pará, from August to October 1999. The epidemiologic data of this research were obtained from the patients' charts and from information obtained at clinical eye examination. All patients in this study were considered cured of Hansen's disease according to the Health Ministery criteria of cure. Results: In 114 eyes $(99.1 \%)$ ocular involvement was observed, the majority (77.2\%) being the virchowian form. The most frequent ocular findings in the adnex were parcial madarosis $(70.4 \%)$ and parcial eyebrow madarosis $(59.1 \%)$. The most frequent ocular finding related to Hansen's disease was the decrease and/or absence of corneal sensitivity observed in $42.6 \%$ of the eyes, followed by paralytic midriasis $(16.5 \%)$ found in the patients after surgery for cataract extraction; this finding was closely related to iris atrophy (8.7\%), commonly found in these patients. Conclusion: The high prevalence of ocular involvement in Hansen patients, especially in virchowian form, indicates the need of complete assistance to these patients even after the resolution of the disease.

Keywords: Leprosy; Leprosy, lepromatous; Mycobacterium leprae; Ocular manifestations; Eye infections, bacterial; Eye diseases/prevention \& control; Hospitals; Cross-sectional studies

\section{REFERÊNCIAS}

1. Cristofolini L, Axcar SR, Vieth H. Ações da enfermagem nos comprometimentos oculares na hanseníase. Salusvita. 1986;5(1):37-51.

2. Dana MR, Hochman MA, Viana MAG, Hill CH, Sugar J. Ocular manisfestations of leprosy in noninstitutionalized community in the United States. Arch Ophthalmol. 1994;112(5):626-9. Erratum in: Arch Ophthalmol. 1995;113(1):24.

3. Oliveira HL, Silva JL, Manso PG, Botene IM, Sartori MB. Envolvimento ocular na hanseníase: estudo em pacientes de ambulatório. Arq Bras Oftalmol. 1996;59(2):162-6

4. Campos WR, Oréfice F. Importância da hanseníase em oftalmologia. J SBU. 1997;1(1).

5. Frota EA, Oréfice F. Lente intra-ocular em portadores de Hansen. Rev Bras Oftalmol. 1989;48(4):205-7.

6. Oréfice F, Miranda D, Boratto LM. Encontro de bacilos da doença de Hansen na conjuntiva, corpo vítreo e retina em um olho enucleado de paciente portador da forma virchowiana. Arq Bras Oftalmol. 1990;53(1):17-9.

7. Santos PM, Maradei J, Barra JMM, Santos RCR, Abreu MT. Estudo das alterações oculares em paciente de hanseníase de hospital-colônia. Arq Bras Oftalmol. 1995;58(2):130-7.

8. Talhari S, Neves RG. Dermatologia tropical - Hanseníase. $3^{\mathrm{a}}$ ed. Manaus (AM): Gráfica Tropical; 1997.

9. Malla OK, Brand F, Anten GF. Ocular findings in leprosy patients in an institution in Nepal (KhoKana). Br J Ophthalmol. 1981;65(4):226-30.

10. Oréfice F, Boratto LM. Estudo da clínica ocular em hansenianos em hospital de dermatologia sanitária: a prevenção em nossas mãos. Arq Bras Oftalmol. 1990; 53(1):13-7.

11. Guimarães FC, Cohen JM, Cruz AAV. Alterações palpebrais na hanseníase de longa duração [abstract]. Arq Bras Oftalmol. 1996;59(4):379.

12. Campos WR, Oréfice F, Sucena MA, Rodrigues CAF. Biópsia conjuntival em pacientes portadores de hanseníase. Rev Bras Oftalmol. 1990;49(3):200-4.

13. Ffytche TJ. Role of iris changes as a cause of blindness in lepromatous leprosy. Br J Ophthalmol. 1981;65(4):231-9.

14. Costa MS, Gallo MEN, Nery JA, Costa BE. Avaliação oftalmológica em hanseníase multibacilar. Arq Bras Oftalmol. 1999;62(6):701-3. 